\title{
Correction to: Frequency and predictors of health services use by native Hawaiians and Pacific islanders: evidence from the U.S. National Health Interview Survey
}

Marie-Rachelle Narcisse ${ }^{1}$, Holly Felix², Christopher R. Long ${ }^{1}$, Teresa Hudson ${ }^{3}$, Nalin Payakachat ${ }^{4}$, Zoran Bursac ${ }^{5}$ and Pearl A. McElfish ${ }^{1 *}$

Correction to: BMC Health Services Research (2018) 18:575

https://doi.org/10.1186/s12913-018-3368-3

Following publication of the original article [1], the author reported the following errors in Table 1 and Table 2.

Table 1 - The numbers in the first row labeled 'Non-Users' should read "81.6" and "79.1-83.9" instead of "816" and "791-839".

Table 2 - In the row labeled "High school/GED" under the "Model 2: Predisposing, Enabling, and Perceived Needs " heading, two of the three cells contain inaccurate results as mentioned below:

\section{Incorrect values:}

Single vs. Non-Users:

$$
1.088
$$

Multiple vs. Single:

1.067

(0.721-1.581)

\section{Correct values:}

Single vs. Non-Users:

1.061

$(0.721-1.563)$
Multiple vs. Single:

$(0.815-1.345)$

\begin{abstract}
Author details
${ }^{1}$ Office of Community Health and Research, University of Arkansas for Medical Sciences Northwest, 1125 North College Ave, Fayetteville, AR 72703, USA. ${ }^{2}$ Fay W. Boozman College of Public Health, University of Arkansas for Medical Sciences, 4301 West Markham St, Little Rock, AR 72205, USA. ${ }^{3}$ Division of Health Services Research, University of Arkansas for Medical Sciences, 4301 West Markham St, Little Rock, AR 72205, USA. ${ }^{4}$ Division of Pharmaceutical Evaluation and Policy, University of Arkansas for Medical Sciences, 4301 West Markham St, Little Rock, AR 72205, USA. ${ }^{5}$ Division of Biostatistics, University of Tennessee Health Science Center, 910 Madison Ave, Memphis, TN 38163, USA.
\end{abstract}

Published online: 04 December 2018

\section{Reference}

1. Narcisse M-R, et al. Frequency and predictors of health services use by native Hawaiians and Pacific islanders: evidence from the U.S. National Health Interview Survey. BMC Health Serv Res. 2018;18:575. https://doi.org/ 10.1186/s12913-018-3368-3.

* Correspondence: pamcelfish@uams.edu

${ }^{1}$ Office of Community Health and Research, University of Arkansas for Medical Sciences Northwest, 1125 North College Ave, Fayetteville, AR 72703, USA

Full list of author information is available at the end of the article

(c) The Author(s). 2018 Open Access This article is distributed under the terms of the Creative Commons Attribution 4.0 International License (http://creativecommons.org/licenses/by/4.0/), which permits unrestricted use, distribution, and reproduction in any medium, provided you give appropriate credit to the original author(s) and the source, provide a link to the Creative Commons license, and indicate if changes were made. The Creative Commons Public Domain Dedication waiver (http://creativecommons.org/publicdomain/zero/1.0/) applies to the data made available in this article, unless otherwise stated. 
Table 1 Describing NHPIs' Health Services Use based on Andersen's Model

\begin{tabular}{|c|c|c|}
\hline$n=2172$ & Percentages or Means ${ }^{a}$ & 95\% Confidence Intervals or Standard Errors \\
\hline \multicolumn{3}{|l|}{ Health care utilization: Outcomes } \\
\hline \multicolumn{3}{|l|}{ ED/ER visits } \\
\hline Non-Users & 81.6 & $79.1-83.9$ \\
\hline Single-users (1 visit/year) & 11.4 & $9.6-13.5$ \\
\hline Multiple-users ( $\geq 2$ visits/year) & 7.0 & $5.9-8.4$ \\
\hline \multicolumn{3}{|l|}{ Outpatient services } \\
\hline Non-Users & 26.6 & $24.0-29.3$ \\
\hline Occasional-users (1-3 visits/year) & 46.1 & $42.8-49.4$ \\
\hline Moderate-users (4-9 visits/year) & 17.0 & $14.9-19.3$ \\
\hline Frequent-users ( $\geq 10$ visits/year) & 10.4 & $8.5-12.6$ \\
\hline \multicolumn{3}{|l|}{ Predisposing factors } \\
\hline Age (mean) & 40.8 & $39.9-41.7$ \\
\hline Females & 50.2 & $46.2-54.2$ \\
\hline \multicolumn{3}{|l|}{ Education level } \\
\hline Less than high school & 10.2 & $8.0-13.0$ \\
\hline High-school/GED & 39.6 & $36.1-43.3$ \\
\hline High-school or more & 50.2 & $47.0-53.8$ \\
\hline Married; living with a partner (yes) & 60.7 & $57.5-63.9$ \\
\hline Unemployed & 33.1 & $28.5-38.0$ \\
\hline Born in the US (yes) & 70.5 & $66.9-73.9$ \\
\hline \multicolumn{3}{|l|}{ Enabling factors } \\
\hline \multicolumn{3}{|l|}{ Poverty level } \\
\hline$<100 \%$ FPL & 15.9 & $12.9-19.5$ \\
\hline 100-199\% FPL & 24.1 & $21.4-27.1$ \\
\hline 200-399\% FPL & 29.8 & $26.5-33.3$ \\
\hline$\geq 400 \% \mathrm{FPL}$ & 30.2 & $28.3-32.2$ \\
\hline Uninsured (yes) & 6.2 & $4.8-8.0$ \\
\hline Usual source of routine/preventive care (yes) & 87.4 & $84.9-89.6$ \\
\hline Use eHealth information (yes) & 19.3 & $17.0-21.9$ \\
\hline Ability to afford health care (mean, standard error) & 0.3 & 0.02 \\
\hline Neighborhood social support (mean, standard error) & 12.4 & 3.1 \\
\hline Barriers to health care access (mean, standard error) & 0.1 & 0.02 \\
\hline \multicolumn{3}{|l|}{ Needs } \\
\hline \multicolumn{3}{|l|}{ Evaluated needs (number of CDs) } \\
\hline 0 & 39.7 & $35.9-43.7$ \\
\hline 1 & 23.9 & $21.3-26.7$ \\
\hline$\geq 2$ & 36.4 & $33.9-38.9$ \\
\hline \multicolumn{3}{|l|}{ Perceived needs (Health Status) } \\
\hline Poor-Fair & 13.7 & $12.3-15.1$ \\
\hline Good & 28.3 & $25.1-31.7$ \\
\hline Very good & 30.0 & $26.8-33.3$ \\
\hline Excellent & 28.0 & $23.9-32.6$ \\
\hline
\end{tabular}

Source: NHPI-NHIS 2014

aWeighted means and percentages. Valid estimates do not account for missing data

Note: FPL: Federal Poverty Level. CD: Chronic Diseases. GED: General Educational Development

ED/ER: Emergency department/Emergency room. US: United States 
Table 2 Predicting use of ED services among NHPIs: Odds-Ratio and Confidence Interval (95\% Cl)

\begin{tabular}{|c|c|c|c|c|c|c|c|}
\hline \multirow[b]{2}{*}{ Predisposing factors } & \multicolumn{3}{|c|}{$\begin{array}{l}\text { Model 1: Predisposing, Enabling, and Evaluated } \\
\text { Needs }\end{array}$} & & \multicolumn{3}{|c|}{$\begin{array}{l}\text { Model 2: Predisposing, Enabling, and Perceived } \\
\text { Needs }\end{array}$} \\
\hline & $\begin{array}{l}\text { Single } \\
\text { vs. Non-Users }\end{array}$ & $\begin{array}{l}\text { Multiple } \\
\text { vs. Non-Users }\end{array}$ & $\begin{array}{l}\text { Multiple } \\
\text { vs. Single }\end{array}$ & & $\begin{array}{l}\text { Single } \\
\text { vs. Non-Users }\end{array}$ & $\begin{array}{l}\text { Multiple } \\
\text { vs. Non-Users }\end{array}$ & $\begin{array}{l}\text { Multiple } \\
\text { vs. Single }\end{array}$ \\
\hline Age & $\begin{array}{l}1.007 \\
(0.997-1.018)\end{array}$ & $\begin{array}{l}1.010 \\
(0.993-1.027)\end{array}$ & $\begin{array}{l}1.002 \\
(0.993-1.011)\end{array}$ & & $\begin{array}{l}1.008 \\
(0.997-1.020)\end{array}$ & $\begin{array}{l}1.015^{*} \\
(1.001-1.030)\end{array}$ & $\begin{array}{l}1.007 \\
(0.998-1.015)\end{array}$ \\
\hline Females & $\begin{array}{l}0.861 \\
(0.627-1.181)\end{array}$ & $\begin{array}{l}0.823 \\
(0.538-1.261)\end{array}$ & $\begin{array}{l}0.957 \\
(0.801-1.143)\end{array}$ & & $\begin{array}{l}0.914 \\
(0.707-1.182)\end{array}$ & $\begin{array}{l}0.852 \\
(0.526-1.383)\end{array}$ & $\begin{array}{l}0.933 \\
(0.731-1.191)\end{array}$ \\
\hline High-school or less & $\begin{array}{l}1.083 \\
(0.564-2.081)\end{array}$ & $\begin{array}{l}1.109 \\
(0.487-2.526)\end{array}$ & $\begin{array}{l}1.024 \\
(0.851-1.232)\end{array}$ & & $\begin{array}{l}1.088 \\
(0.621-1.906)\end{array}$ & $\begin{array}{l}1.161 \\
(0.451-2.988)\end{array}$ & $\begin{array}{l}1.067 \\
(0.721-1.581)\end{array}$ \\
\hline High-school/GED & $\begin{array}{l}1.208 \\
(0.795-1.835)\end{array}$ & $\begin{array}{l}1.277 \\
(0.815-2.000)\end{array}$ & $\begin{array}{l}1.057 \\
(0.901-1.240)\end{array}$ & & $\begin{array}{l}1.061 \\
(0.721-1.563)\end{array}$ & $\begin{array}{l}1.111 \\
(0.590-2.092)\end{array}$ & $\begin{array}{l}1.047 \\
(0.815-1.345)\end{array}$ \\
\hline Married/living with partner & $\begin{array}{l}0.721 \\
(0.423-1.228)\end{array}$ & $\begin{array}{l}0.655 \\
(0.333-1.288)\end{array}$ & $\begin{array}{l}0.908 \\
(0.648-1.273)\end{array}$ & & $\begin{array}{l}0.757 \\
(0.441-1.298)\end{array}$ & $\begin{array}{l}0.609 \\
(0.279-1.329)\end{array}$ & $\begin{array}{l}0.806 \\
(0.566-1.148)\end{array}$ \\
\hline Unemployed & $\begin{array}{l}1.002 \\
(0.652-1.540)\end{array}$ & $\begin{array}{l}1.003 \\
(0.575-1.748)\end{array}$ & $\begin{array}{l}1.001 \\
(0.882-1.135)\end{array}$ & & $\begin{array}{l}0.951 \\
(0.694-1.304)\end{array}$ & $\begin{array}{l}0.915 \\
(0.518-1.616)\end{array}$ & $\begin{array}{l}0.962 \\
(0.742-1.246)\end{array}$ \\
\hline Born in the US & $\begin{array}{l}1.302 \\
(0.841-2.014)\end{array}$ & $\begin{array}{l}1.407 \\
(0.810-2.445)\end{array}$ & $\begin{array}{l}1.081 \\
(0.823-1.420)\end{array}$ & & $\begin{array}{l}1.184 \\
(0.793-1.767)\end{array}$ & $\begin{array}{l}1.349 \\
(0.703-2.590)\end{array}$ & $\begin{array}{l}1.140 \\
(0.841-1.544)\end{array}$ \\
\hline \multicolumn{8}{|l|}{ Enabling factors } \\
\hline$<100 \% \mathrm{FPL}$ & $\begin{array}{l}1.387 \\
(0.705-2.731)\end{array}$ & $\begin{array}{l}1.528 \\
(0.563-4.148)\end{array}$ & $\begin{array}{l}1.101 \\
(0.716-1.695)\end{array}$ & & $\begin{array}{l}1.158 \\
(0.620-2.164)\end{array}$ & $\begin{array}{l}1.297 \\
(0.391-4.304)\end{array}$ & $\begin{array}{l}1.120 \\
(0.621-2.022)\end{array}$ \\
\hline 100-199\% FPL & $\begin{array}{l}1.433 \\
(0.875-2.348)\end{array}$ & $\begin{array}{l}1.593^{*} \\
(1.023-2.482)\end{array}$ & $\begin{array}{l}1.112 \\
(0.829-1.492)\end{array}$ & & $\begin{array}{l}1.128 \\
(0.738-1.726)\end{array}$ & $\begin{array}{l}1.239 \\
(0.633-2.427)\end{array}$ & $\begin{array}{l}1.098 \\
(0.838-1.440)\end{array}$ \\
\hline 200-399\% FPL & $\begin{array}{l}1.003 \\
(0.656-1.533)\end{array}$ & $\begin{array}{l}1.004 \\
(0.580-1.738)\end{array}$ & $\begin{array}{l}1.001 \\
(0.883-1.134)\end{array}$ & & $\begin{array}{l}0.925 \\
(0.645-1.328)\end{array}$ & $\begin{array}{l}0.871 \\
(0.460-1.650)\end{array}$ & $\begin{array}{l}0.942 \\
(0.708-1.254)\end{array}$ \\
\hline Uninsured & $\begin{array}{l}0.920 \\
(0.263-3.224)\end{array}$ & $\begin{array}{l}0.898 \\
(0.166-4.850)\end{array}$ & $\begin{array}{l}0.976 \\
(0.631-1.509)\end{array}$ & & $\begin{array}{l}0.936 \\
(0.369-2.379)\end{array}$ & $\begin{array}{l}0.890 \\
(0.159-4.982)\end{array}$ & $\begin{array}{l}0.950 \\
(0.431-2.097)\end{array}$ \\
\hline Usual source of care & $\begin{array}{l}1.102 \\
(0.425-2.859)\end{array}$ & $\begin{array}{l}1.134 \\
(0.315-4.085)\end{array}$ & $\begin{array}{l}1.029 \\
(0.734-1.443)\end{array}$ & & $\begin{array}{l}1.086 \\
(0.464-2.538)\end{array}$ & $\begin{array}{l}1.157 \\
(0.251-5.329)\end{array}$ & $\begin{array}{l}1.066 \\
(0.538-2.110)\end{array}$ \\
\hline Use eHealth information & $\begin{array}{l}1.520 \\
(0.917-2.519)\end{array}$ & $\begin{array}{l}1.719^{*} \\
(1.080-2.736)\end{array}$ & $\begin{array}{l}1.131 \\
(0.792-1.616)\end{array}$ & & $\begin{array}{l}1.436 \\
(0.912-2.262)\end{array}$ & $\begin{array}{l}1.901 * \\
(1.137-3.180)\end{array}$ & $\begin{array}{l}1.117 \\
(0.873-1.430)\end{array}$ \\
\hline Ability to afford health care & $\begin{array}{l}1.236 \\
(0.990-1.543)\end{array}$ & $\begin{array}{l}1.316 \\
(0.823-2.105)\end{array}$ & $\begin{array}{l}1.064 \\
(0.809-1.400)\end{array}$ & & $\begin{array}{l}1.154 \\
(0.980-1.359)\end{array}$ & $\begin{array}{l}1.290 \\
(0.893-1.864)\end{array}$ & $\begin{array}{l}1.12 \\
(0.873-1.437)\end{array}$ \\
\hline Neighborhood social support & $\begin{array}{l}0.951 \\
(0.884-1.023)\end{array}$ & $\begin{array}{l}0.937^{*} \\
(0.881-0.997)\end{array}$ & $\begin{array}{l}0.985 \\
(0.948-1.025)\end{array}$ & & $\begin{array}{l}0.971 \\
(0.914-1.031)\end{array}$ & $\begin{array}{l}0.948 \\
(0.877-1.026)\end{array}$ & $\begin{array}{l}0.977 \\
(0.949-1.006)\end{array}$ \\
\hline Barriers to health care access & $\begin{array}{l}1.138 \\
(0.809-1.601)\end{array}$ & $\begin{array}{l}1.183 \\
(0.797-1.756)\end{array}$ & $\begin{array}{l}1.039 \\
(0.916-1.179)\end{array}$ & & $\begin{array}{l}1.151 \\
(0.837-1.582)\end{array}$ & $\begin{array}{l}1.283 \\
(0.804-2.048)\end{array}$ & $\begin{array}{l}1.115 \\
(0.916-1.357)\end{array}$ \\
\hline Evaluated needs (CDs) & & & & $\begin{array}{l}\text { Perceived needs } \\
\text { (health status) }\end{array}$ & & & \\
\hline 1 & $\begin{array}{l}1.191 \\
(0.763-1.860)\end{array}$ & $\begin{array}{l}1.254 \\
(0.715-2.199)\end{array}$ & $\begin{array}{l}1.053 \\
(0.862-1.286)\end{array}$ & Good & $\begin{array}{l}0.484^{*} \\
(0.282-0.830)\end{array}$ & $\begin{array}{l}0.275^{* *} \\
(0.107-0.708)\end{array}$ & $\begin{array}{l}0.570 \\
(0.241-1.347)\end{array}$ \\
\hline \multirow[t]{2}{*}{$\geq 2$} & $\begin{array}{l}1.881 \\
(0.971-3.645)\end{array}$ & $\begin{array}{l}2.267^{* *} \\
(1.399-3.672)\end{array}$ & $\begin{array}{l}1.205 \\
(0.714-2.034)\end{array}$ & Very good & $\begin{array}{l}0.369^{* *} \\
(0.202-0.674)\end{array}$ & $\begin{array}{l}0.170^{* * *} \\
(0.074-0.391)\end{array}$ & $\begin{array}{l}0.462 \\
(0.161-1.324)\end{array}$ \\
\hline & & & & Excellent & $\begin{array}{l}0.451^{* *} \\
(0.254-0.801)\end{array}$ & $\begin{array}{l}0.243^{* *} \\
(0.090-0.659)\end{array}$ & $\begin{array}{l}0.540 \\
(0.212-1.374)\end{array}$ \\
\hline
\end{tabular}

